\title{
SMOOTH EMBEDDINGS OF HOMOLOGICALLY SIMILAR MANIFOLDS
}

\author{
BY
}

\author{
DENNIS M. ROSEMAN $\left({ }^{1}\right)$
}

\begin{abstract}
We consider the situation where we have two smooth $n$-manifolds $N \subseteq M$ with $H_{*}(M, N)=0$ and show that given a smooth embedding of $N$ into some manifold $Q$ we may, under suitable conditions, extend this to embeddings of $M$ into $Q, Q \times I$, or $Q \times I^{2}$ (where $I$ is the unit interval). We can apply these results to obtain smooth embeddings of homologically $k$-connected manifolds into $(2 n-k+1)$-dimensional euclidian space.
\end{abstract}

0. Preliminaries. We will be primarily interested in smooth manifolds with (perhaps empty) boundary, by which we will mean a manifold with a $C^{\infty}$ structure. If $M$ is a smooth $n$-dimensional manifold we will sometimes use the notation $M^{n}$ if we need to emphasize the dimension. If $f: M^{k} \rightarrow N^{n}$ is a smooth embedding of $M$ into $N$, then we will frequently without explicit mention make the natural identification of $M$ and $f(M)$ in order to avoid excessive notation. All manifolds and maps will be smooth unless otherwise stated. If we are given an embedding of a manifold $N$ in a manifold $M$, we can always change the embedding slightly so that $N$ will be contained in the interior of $M$ by shrinking $N$ away from the boundary via the collar neighborhood of $\partial M$ in $M$.

1. Statement and discussion of main results. The problem which we will be most concerned with is the following. Suppose that we have two smooth $n$-manifolds with boundary $M^{n}$ and $N^{n}$ with $N \subseteq M$ with $H_{*}(M, N)=0$. Then it would seem that $M$ and $N$ must be quite similar in many ways. We might expect for example that if we could embed $N$ smoothly into some smooth manifold $Q$ that we could also embed $M$ into $Q$. We will shortly give a counterexample to this conjecture, yet we will be able to prove some theorems that are very close to this. We will generally assume that $M, N, \partial M, \partial N$ are connected.

The following three theorems are our main results along these lines. Let Hypothesis (\#) denote " $M^{n}$ and $N^{n}$ are smooth manifolds with boundary with $N \subseteq M, H_{*}(M, N)=0$, and $n>4$."

Presented to the Society, January 24, 1969; received by the editors August 6, 1969 and, in revised form, March 9, 1971 and November 1, 1971.

AMS (MOS) subject classifications (1970). Primary 57D40, 57D90; Secondary 57D65.

Key words and phrases. Homology $n$-connected, $h$-cobordism, homology cobordism, handle decomposition.

(1) Portions of this paper submitted in partial fulfillment of the requirements for the Doctor of Philosophy degree in the Graduate School, University of Michigan. 
Theorem A. Given Hypothes is (\#), if there is a smootb 1-trivial embedding, $f: N \rightarrow Q^{q}$ (i.e., an embedding such that $f_{\#}: \pi_{1}(N) \rightarrow(0) \subseteq \pi_{1}(Q)$ ) and if $n<q$, then there is a smooth embedding $M \times I \subseteq Q \times I^{2}$.

Theorem B. Given Hypothesis (\#), if there is a smooth 1 -trivial embedding $N \times I \subseteq Q^{q}$, with $q-n>3$ then we can smootbly embed $M \times I \subseteq Q$.

An additional assumption will allow us to obtain better results. Let Hypothesis (\#\#) denote " $M^{n}$ and $N^{n}$ are smooth manifolds with boundary with $N \subseteq M, H_{*}(M, N)=0, n>5$ such that the map $j_{\#}: \pi_{1}(\partial M) \rightarrow \pi_{1}(\overline{M-N})$ induced by the inclusion is onto."

Theorem $\mathbf{A}^{\prime}$. Given Hypothesis (\#\#), then if there is a smooth 1-trivial embedding $N \subseteq Q^{q}$ with $n<q$, then there is a smooth embedding $M \subseteq Q \times I$.

Theorem B'. Given Hypothesis (\#\#), then if there is a smooth 1-trivial embedding $N \subseteq Q^{q}$, with $q-n>3$, then we can smoothly embed $M \subseteq Q$.

Example 1. In general, it is false that if $H_{*}(M, N)=0$ and $N \subseteq E^{q}$, then $M \subseteq E^{q}$. For the example, let $P^{n}$ be a homology sphere which is not simply connected. Let $D^{n}$ be a closed $n$-ball in the interior of $P$, let $M=\overline{P-D}$; let $N$ be a closed $n$-ball in the interior of $M$. Certainly we have $H_{*}(M)=0$; since $N$ is an $n$-ball, $N \subseteq E^{n}$. But $M$ does not embed in $E^{n}$, for if so then we would have an embedding of $S=\partial M$ in $E^{n}$, this sphere would bound a ball in $E^{n}$, and we would be forced to conclude that $M$ was a ball, which it is not. Thus the codimension zero version of the conjecture is false; however, we have no such counterexamples in other low codimensions.

Suppose we have $N^{n} \subseteq M^{n}$ with $H_{*}(M, N)=0$ (we may assume that $N \subseteq$ Int $M$ ). What we really need to examine is the manifold $H=\widehat{M-N}$. By excision, we will have $H_{*}(H, \partial N)=0$ and by duality (Milnor [11]) we will have $H_{*}(H, \partial M)=0$. Now if it happened that $H$ were in fact an $b$-cobordism then the embedding problem is trivial, for if the Whitehead torsion $t(H, \partial M)=a$, then as in Stallings [13] we can construct another $b$-cobordism $H^{\prime}$, with one boundary component $\partial M$ such that $t\left(H^{\prime}, \partial M\right)=-a$. We then form the manifold $M^{\prime}=M+H^{\prime}=N+H+H^{\prime}$. But $H+H^{\prime}$ is an $b$-cobordism with $t\left(H+H^{\prime}, \partial N\right)=0$ and thus $H+H^{\prime} \approx \partial N \times I$; therefore $M^{\prime} \approx N$. Since we can embed $N$ we have an embedding of $M^{\prime}$, but since $M \subseteq M^{\prime}$ this gives an embedding of $M$.

Now if $H$ and each component of the boundary of $H$ were simply connected then it would easily follow that $H$ would be an $b$-cobordism, but if they are not all simply connected then $H$ need not be an $b$-cobordism. The following two examples are useful to keep in mind for relating properties of such manifolds $H$. 
Example 2. Take $H=\overline{M-N}$ where $M$ and $N$ are as in the above example; $M$ is a Poincaré sphere minus a ball; $N$ is a subball of $M$. Then $H$ is an example where both components of $\partial H$ are simply connected (they are spheres) but $H$ is not an $b$-cobordism, since $H$ is not simply connected.

Example 3. Let $M^{n}$ be a Mazur manifold [9]-that is, a contractable manifold with nonsimple connected boundary; let $N$ be an $n$-ball in the interior of $M$. In this case, $H$ and one of the boundaries, $\partial N$, are simply connected, but the other boundary component, $\partial M$, is not.

Example 4. We now give an example of an $H$-cobordism $\left(H, M_{0}, M_{1}\right)$ such that $\pi_{1}\left(M_{0}\right) \approx \pi_{1}(H)$ and $\pi_{1}\left(M_{1}\right) \approx \pi_{1}(H)$ via inclusion and yet $H$ is not an $b$-cobordism. We will use an example of Stallings found in Kervaire [7, Theorem $\mathrm{V}$ ], of an embedding, $f$, of an $n$-sphere, $n \geq 3$, in an $(n+2)$-sphere with $\pi_{1}\left(S^{n+2}-f\left(S^{n}\right)\right) \approx Z$ and $\pi_{2}\left(S^{n+2}-f\left(S^{n}\right)\right) \neq 0$. Let $\gamma$ be the generator of $\pi_{1}\left(S^{n+2}-f\left(S^{n}\right)\right)$. We may represent $\gamma$ by a smoothly embedded circle $g: S^{1} \rightarrow$ $S^{n+2}$. Both $g\left(S^{1}\right)$ and $f\left(S^{n}\right)$ have trivial normal disk bundles-call these $T_{1}$ and $T_{n}$, respectively; we may choose these so that $T_{1} \cap T_{n}=\varnothing$. Note that $\partial T_{1} \approx \partial T_{n} \approx S^{1} \times S^{n}$. Now let $H=\overline{S^{n+2}-T_{1}-T_{n}}$, and let $M_{0}=\partial T_{1}, M_{1}=\partial T_{n}$.

The following three theorems give some indication of what implications follow from the assumption that $M^{n} \supseteq N^{n}$ is a homotopy equivalence. The proofs are straightforward; the crossing with $I^{2}$, in 1.1 , or the crossing with $I$ together with the hypotheses about the fundamental groups in 1.2, is used to assure codimension 3 spines of the manifolds in question so as to apply Lemma 2.10 for the fundamental groups.

Theorem 1.1 (crossing with $I^{2}$ ). Suppose that $M^{n} \supseteq N^{n}, n>3$, is a bomotopy equivalence. Let $M^{\prime}=M \times I^{2} ; N^{\prime}=N \times I^{2}$. We may consider $N^{\prime} \subseteq$ Int $M^{\prime}$; let $H^{\prime}=\overline{M^{\prime}-N^{\prime}}$. Then $H^{\prime \prime}$ is an $b$-cobordism between $\partial M^{\prime}$ and $\partial N^{\prime}$.

Theorem 1.2 (crossing with I). Suppose that $N^{n} \subseteq M^{n}$ is a bomotopy equivalence with $n>4$. Let $M^{\prime}=M \times I ; N^{\prime}=N \times I$, we can consider $N^{\prime} \subseteq$ Int $M^{\prime}$. Let $H^{\prime}=\overline{M^{\prime}-N}, H=\overline{M-N}$, and suppose that $\pi_{1}(\partial M) \rightarrow \pi_{1}(H)$ and $\pi_{1}(\partial N) \rightarrow$ $\pi_{1}(H)$ induced by inclusion are all onto, then $H^{\prime}$ is an b-cobordism.

Theorem 1.3. (a) If $N^{n} \subseteq M^{n}$ is a bomotopy equivalence, $H=\overline{M-N}$, and if $N$ and $\partial N$ are simply connected, then $2 H$ is an b-cobordism. (b) If $N^{n} \subseteq M^{n}$ is a bomotopy equivalence with $N$ simply connected, $N^{\prime}=N \times I, M^{\prime}=M \times I$, $H^{\prime}=\overline{M^{\prime}-N^{\prime}}$, then $2 H^{\prime}$ is an b-cobordism, where $2 H^{\prime}$ denotes two copies of $H^{\prime}$ identified along $\partial M$.

We can now use these theorems to obtain the following smooth embedding results. 
Theorem 1.4. (1) If we have the bypothes is of Theorem 1.1 with $n>3$; then if $N \times I^{2} \subseteq Q$, then $M \times I^{2} \subseteq Q$.

(2) If we have the hypothes is of Theorem 1.2 with $n>4$; then if $N \times I \subseteq Q$. then $M \times I \subseteq Q$.

(3) If we have the hypothes is of Theorem 1.3(a) with $n>4$; then if $N \subseteq Q$, then $M \subseteq Q$. If we have the bypothes is of Theorem 1.3(b) with $n>4$; then if $N \times I \subseteq Q$, then $M \times I \subseteq Q$.

Definition. $\left(H ; M_{0}, M_{1}\right)$ will be called an $H$-cobordism, or homology cobordism if $\partial H=M_{0} \cup M_{1}$ with $M_{0} \cap M_{1}=\varnothing$, and such that $H_{*}\left(H, M_{0}\right)=0$. (Note that is follows from duality that $H_{*}\left(H, M_{0}\right)=0$ implies that $H_{*}\left(H, M_{1}\right)=0$.)

2. Handlebody theorems. Suppose we write $D^{n}$ as $D^{r} \times D^{n-r}$, and consider $\partial D^{r} \times D^{n-r}$ to be a subset of $\partial D^{n}$ via the formula: $\partial D^{n}=\partial D^{r} \times D^{n-r}+D^{r} \times$ $\partial D^{n-r-1}$ where the identifications are on $\partial D^{r} \times \partial D^{n-r-1}$. Suppose also that $N^{n}$ is a given smooth $n$-manifold and that $A$ is a smooth submanifold of $\partial N$ which is diffeomorphic to $\partial D^{r} \times D^{n-r}$ via a diffeomorphism $h, b: \partial D^{r} \times D^{n-r} \rightarrow$ $A$. The map $b$ is called the $a$-map or attaching map. The space $N+D^{n}$ with identifications via $b$ will be a smooth manifold called $N$ plus the $r$-bandle $b$; this will usually be denoted more simply by $N+b$. If the value of $r$ is to be emphasized, we will also call $N+b, N$ plus a bandle of type $r$.

The subset $A$ will be called the attaching set of $b$, or the $a$-set of $b$. The subset of $A$ corresponding to $\partial D^{r} \times(0)$ will be called the attaching sphere of $b$, or the $a$-sphere. The subset of $\partial(N+b)$ corresponding to $D^{r} \times \partial D^{n-r}$ will be called the boundary of $h$, and denoted $\partial h$. A subset corresponding to a subset of $\partial b$ of the form $x \times \partial D^{n-r}$, where $x \in D^{r}$, will be called a $b$-sphere of $b$.

Definition. 'A bandle decomposition of $M$ relative to $N$, where $M$ and $N$ are both $n$-manifolds will be a diffeomorphism of $M$ with $N$ plus some handles; we will write this as $M=N+b_{1}+b_{2}+\cdots+b_{s}$. If we wish to emphasize the types of the handles in the decomposition, we will use superscripts so that, for example, $N+b_{1}^{i}+b_{2}^{j}$ will denote $N$ plus a particular handle of type $i$ plus a particular handle of type $j . M^{n}$ is a bandle decomposition relative to $N^{n-1}$ will mean that $M$ has a handle decomposition on $N \times I$ with all handles attached on $N \times(1)$.

Lemma 2.1. Given a bandle decomposition $M=N+b_{1}+\cdots+b_{s}$, then there is a natural bandle decomposition on $M \times I$ that bas bandles of the same type as those in the decomposition of $M$. That is, we may write $M \times I=$ $N \times I+g_{1}+\cdots+g_{s}$ where if $A_{i}$ and $S_{i}, i=1, \cdots, s$, denote the a-set and the a-sphere of $b_{i}$, then $A_{i} \times I$ and $S_{i} \times 1 / 2$ will correspond to the a-set and a-sphere of $g_{i}$. 
Proposition 2.2 (changing the order of the handles if the a-sets are disjoint). Suppose that $M=N+b+b^{\prime}$; with $A$ and $A^{\prime}$ denoting the a-sets of $b$ and $b^{\prime}$, respectively. If $A$ and $A^{\prime}$ are disjoint subsets of $\partial N$, then $N+b+b^{\prime} \approx N+$ $b^{\prime}+b$.

The following is a standard theorem about handlebodies [6].

Lemma 2.3 (moving an $a$-sphere by an isotopy). Suppose that $M=N+b_{1}+$ $\cdots+b_{s}$ is a bandle decomposition and that $S$ denotes the a-sphere of $b_{1}$. Suppose that we are given an isotopy $H_{t}$ of $S$ in $\partial N$. Then we can obtain an equivalent bandle decomposition $M=N+g_{1}+\cdots+g_{s}$ such that the a-sphere of $g_{1}$ is $H_{1}(S)$.

The following lemma is a version of the product neighborhood theorem.

Lemma 2.4. If $P$ and $Q$ are smooth manifolds and $b^{\prime}: P \times\{0\} \rightarrow Q \times\{0\}$ is a diffeomorphism then there is a diffeomorphism, $b$, unique up to isotopy, $b: P \times I \rightarrow Q \times I$ such that $b \mid P \times\{0\}=b^{\prime}$.

Definition. Suppose what we are given a handle decomposition $M^{n}=N^{n}+$ $b_{1}+b_{2}+\cdots+b_{s}$. Then we will say that the decomposition is nicely bandled if

(1) The handles are added in order of increasing type-that is, if $i \leq j$ then the type of $b_{i}$ is less than or equal to the type of $b_{j}$.

(2) Let $N(k)$ denote $N$ plus all those handles of type less than or equal to $k(N(k)$ is analogous to the $k$-skeleton). We will require that the $a$-sets of all the $(k+1)$-handles in the decomposition are disjoint subsets of $\partial N(k)$, for all values of $k$.

(3) If a $(k+1)$-handle intersects a $k$-handle we will require that the $a$-set of the $(k+1)$-handle goes right around the $k$-handle. By this we mean the following. Suppose that $b$ is a $k$-handle in the decomposition, that $A^{\prime}$ is the $a$-set of $b^{\prime}$, and that $A \cap \partial b \neq \varnothing$. We have $A^{\prime} \approx \partial D^{k+1} \times D^{n-k-1}$. We will require that $A^{\prime} \cap \partial b$ consists of a disjoint collection of $(n-1)$-disks denoted by $D_{i}$, each corresponding to a subset of the form $B_{i}^{k} \times D^{n-k-1}$ where the $B_{i}$ are subdisks of $\partial D^{k+1}$. Furthermore, this cartesian structure must be compatible with the cartesian structure of $\partial b \approx D^{k} \times \partial D^{n-k}$. That is, there is a collection of $(n-k-1)$-disks in $\partial D^{n-k},\left\{C_{i}\right\}$, such that if $f_{i}: D^{k} \rightarrow \partial D^{k+1}$ is the inclusion map of $B_{i}$ and if $g_{i}: D^{n-k-1} \rightarrow \partial D^{n-k}$ is the inclusion map of $C_{i}$, then the inclusion of $D_{i}$ into $\partial b$ is given by $f_{i} \times g_{i}$.

Remarks. Conditions (1) and (2) are essentially the requirements of a "nice" handle decomposition in the sense of Smale [14]-i.e., one corresponding to a "nice" or self-indexing Morse function.

Condition (3) essentially says that the set $A^{\prime}$ does not double back on the 
handle $b$, nor does it twist around $b$; furthermore the "fibers of $A^{\prime}$ line up with the fibers of $\partial b . "$

The proof of the following theorem is an easy generalization of the proof that every handle decomposition is equivalent to a nice handle decomposition, Barden [1].

Theorem 2.5. Let $M=N+b_{1}+\cdots+b_{s}$ be a bandle decomposition. Then there is an equivalent bandle decomposition of $M$ relative to $N$ which is nicely bandled.

In the constructions which are to follow, we will need the following concept of relative transversality.

Definition. Suppose that $A^{a}, B^{b}$ and $C^{c}$ are submanifolds of $Q^{q}$, with $C \subseteq A \cap B$. We will consider the tangent manifolds of $A$ and $B$ to be contained in the tangent manifold of $Q$, thus $T(A)_{x}$ will denote the tangent plane of $A$ at $x$ which we will consider as a hyperplane in $T(Q)_{x}$, the tangent plane of $Q$ at $x$. We will say that $A$ is transverse to $B$ relative to $C$ if the following hold:

(1) If $x \in C$, then $T(A)_{x}$ and $T(B)_{x}$ span an $(a+b-c)$-dimensional hyperplane in $T(Q)_{x}$.

(2) If $x \notin C$, then we require that $A$ and $B$ are transverse in the usual sense; that is, that $T(A)_{x}$ and $T(B)_{x}$ span $T(Q)_{x}$, it being understood that this condition is vacuous if $x \notin A \cap B$, and that if $a+b<q$, then transversality at $x$ will mean that $x \notin A \cap B$.

Lemma 2.6. Suppose that $M^{n}=N^{n}+b$, where $b$ is a $(k+1)$-bandle; and suppose we have $N^{n} \subseteq Q^{q}$. Let $A$ and $S$ be the a-set and a-sphere, respectively, of the bandle $b$. Then we can extend the embedding of $N$ to an embedding of $M$ if and only if

(1) there is a $(k+1)$-disk $B$ in $Q$ with $B \cap N=S=\partial B$ and $B$ is trans. verse to $A$ (and therefore to $N$ ) relative to $S$, and

(2) a certain obstruction a is zero. This obstruction is an element in $\pi_{k}(V(q-k-1, n-k-1))$.

Proof. The proof of the lemma follows easily after we define $\alpha$. We first define a map $F: \partial B \rightarrow V(q-k-1, n-k-1)$ as follows. By our transversality, we may assume $B$ is orthogonal to $A$. For each $x \in \partial B$, let $f(x)$ be the $(n-k-1)$-frame at $x$, normal to $B$, corresponding to the standard frame of $D^{n-k-1}$ via $\{x\} \times D^{n-k-1} \subseteq A \approx \partial B \times D^{n-k-1}$; this may be considered a frame in $R^{q-k-1}$ by projection on the $(q-k-1)$-dimensional fiber of the trivial normal bundle of $B$ in $Q$. Then $a$ will be the homotopy class of $f$ in $\pi_{k}(V(q-k-1, n-k-1))$. 
Lemma 2.7. If $q-n>k$, then $\pi_{k}(V(q-k-1, n-k-1))=0$.

Proof. By Steenrod $\left[15,25.6\right.$, p. 132], we find that $\pi_{k}(V(x, y))=0$ if $x-y>k$.

Combining the above two lemmas we have

Lemma 2.8. If we have $M^{n}=N^{n}+b$, where $b$ is a $k$-bandle, and suppose that $N^{n} \subseteq Q^{q}$, and suppose there is a $k$-disk $B$, in $Q$ which spans $S$, the set corresponding to the a-sphere of $b$, then we may find a smootb subdisk $B^{\prime}$ in $Q$ which spans $S$ and which is transverse to $N$ relative to $S$ if $q>n+k$.

Definition. Suppose that $S^{a}$ and $S^{b}$ are transverse subspheres of $Q^{a+b}$; and suppose that we choose orientations of each of these manifolds. An orientation will give a specific orientation to each tangent plane, given say by a preferred ordered basis. Then if $x \in S^{a} \cap S^{b}$, the intersection number will be defined to be plus one if we take a basis of $T(Q)_{x}$ by taking first the basis vectors which correspond to the chosen basis of $T\left(S^{a}\right)_{x}$ in $T(Q)_{x}$ and then the preferred basis vectors corresponding to the chosen basis of $T\left(S^{b}\right)_{x}$, and if this ordered basis gives the same orientation to $T(Q)_{x}$ as the chosen one. If the orientation is not the chosen one, then we will say the intersection number of the point $x$ is a minus one.

If $b$ is a $k$-handle, and $b^{\prime}$ is a $(k+1)$-handle in some handle decomposition, then the intersection number of these two handles will be the algebraic sum of all the intersection numbers of the $a$-sphere of the $(k+1)$-handle and the $b$-spher of the $k$-sphere. The sign of the intersection number will depend on the arbitrary choices of orientations of the manifolds involved.

Definition. A handle decomposition will be called an $b$-decomposition if $M=N+b_{1}+\cdots+b_{s}+k_{1}+\cdots+k_{s}$ where the $b_{i}$ are all $k$-handles and the $k_{i}$ are all $(k+1)$-handles, with the decomposition nicely handled, where we require that the intersection number of $b_{i}$ and $k_{i}$ is one, and that the intersection number of $b_{i}$ and $k_{j}$ for $i \neq j$ is zero. We will say that such a handle decomposition is an b-decomposition of type $(k, k+1)$.

Theorem 2.9 (the $b$-decomposition theorem). Suppose that $M^{n} \supseteq N^{n}$ with $H_{*}(M, N)=0$, with $\partial N$ and $\partial M$ connected and $n>3$, then $M$ can be written as " $N$ plus a sum of b-decompositions"; that is, we may write

$$
\begin{aligned}
M= & N+b_{1}^{1}+\cdots+b_{s_{1}}^{1}+k_{1}^{2}+\cdots+k_{s_{1}}^{2} \\
& +b_{1}^{2}+\cdots+b_{s_{2}}^{2}+k_{1}^{3}+\cdots+k_{s_{2}}^{2}+b_{1}^{n-2}+\cdots+b_{s_{n-2}^{n-2}}^{n-k_{1}^{n-1}+\cdots+k_{s_{n-2}}^{n-1}}
\end{aligned}
$$

where, if we let $N(j-1)=N+b_{1}^{1}+\cdots+k_{s_{j-1}}^{j}$ (i.e., $N(j-1)$ is $N$ plus all the bandles of type j-1 or less, plus all the j-handles of the type denoted by $k_{1}^{j}$ ) 
then for each $j$, we have $\sum_{i=1}^{s_{j}} h_{i}^{j}+\sum_{i=1}^{s_{j}} k_{i}^{j+1}$ is an b-decomposition on $N(j-1)$.

Proof. $(M, N)$ has some relative handle decomposition, say, $M=N+g_{1}^{1}+$ $\cdots+g_{r}^{n-1}$; we will refer to this decomposition as $\mathcal{D}$. We will show that we can find an equivalent handle decomposition with the desired properties.

Let $C_{*}(M, N)$ be an associated algebraic relative $C W$ complex associated with $\mathcal{D}$. This will have one $r$-cell for each $r$-handle. Let $\partial_{r}$ denote the boundary operator, $\partial_{r}: C_{r} \rightarrow C_{r-1}$. Let $Z_{r}=\operatorname{ker} \partial_{r}$, and let $B_{r-1}=\partial_{r}^{r}\left(C_{r}\right)$. Then we have an exact sequence:

$$
0 \rightarrow Z_{r} \stackrel{\text { inc }}{\longrightarrow} C_{r} \stackrel{\dot{\partial}_{r}}{\longrightarrow} B_{r-1} \rightarrow 0
$$

Thus we may write $C_{r}=Z_{r} \oplus D_{r}$ where if we let $\partial_{r}^{\prime}=\partial_{r} \mid D_{r}$, then $\partial_{r}^{\prime}: D_{r} \rightarrow$ $B_{r-1}$ is an isomorphism. However, since we have $H_{*}\left(C_{*}\right)=0, B_{r-1}=Z_{r-1}$, we may also think of this as $\partial_{r}^{\prime}: D_{r} \approx Z_{r-1}$.

We will prove the following statement by induction, on $m$.

Statement $S_{m}$. There is a handle decomposition, equivalent to $\mathfrak{D}$, such that $M=N+b_{1}^{1}+\cdots+k_{s_{m-1}}^{m}$ plus some additional handles $\left\{g_{i}^{j}\right\}$ such that if $z_{i}^{j}$ is the generator of $C_{j}$ corresponding to the handle $b_{i}^{j}$, and if $d_{i}^{j}$ is the generator of $C_{j}$ corresponding to the $k_{i}^{j}$, then for all $k \leq m$ we have $\left\{z_{i}^{k}\right\}^{i}$ generates $Z_{k}$ and $\left\{d_{i}^{k}\right\}$ generates $D_{k}$ and $\partial d_{i}^{k}=\partial_{r}^{\prime}\left(d_{i}^{k}\right)=z_{i}^{k-1}$. (Of course, $\partial z_{i}^{k}=0 ;$ the $z_{i}^{k}$, s are cycles.)

The theorem we wish to prove is $S_{n-1}$.

Proof of $S_{1}$. We have no zero handles in our decomposition, thus no zero cells in the relative $C W$ complex, and so $C_{1}=Z_{1}$; and we simply choose the $\left\{z_{i}^{1}\right\}$ to correspond to the generators of the handles $\left\{g_{i}^{1}\right\}$ in the decomposition $\mathscr{D}$; there will be no $\left\{d_{i}^{1}\right\}$.

Proof of $S_{m}$ for $m \leq n-2$, assuming $S_{m-1}$. We have two bases for $C_{i}$. One will be the basis determined by the $i$-handles of the handle decomposition obtained in $S_{i-1}$, this will be denoted by $\left\{c_{j}^{i}\right\}$, these will be the $\left\{g_{j}^{i}\right\}$ s. The second basis for $C_{i}$ will be denoted by $\left\{e_{j}^{i}\right\}$ where $\left\{e_{j}^{i}\right\}=\left\{d_{j}^{i}\right\} \cup\left\{z_{j}^{i}\right\}$. Here $\left\{z_{j}^{i}\right\}$ is an arbitrarily chosen basis for $Z_{i}$, and we define $d_{j}^{i}$ by $d_{j}^{i}=\left(\partial_{i}^{\prime}\right)^{-1}\left(z_{j}^{i-1}\right)$.

Let $A$ be the matrix relating the basis $\left\{c_{j}^{i}\right\}$ to the basis $\left\{e_{j}^{i}\right\}$; that is, the $j$ th column of the matrix $A$ is the coordinate of $c_{j}^{i}$ with respect to the basis $\left\{e_{j}^{i}\right\}$.

Since $A$ is an invertible matrix, it can be reduced to the identity matrix by elementary column operations.

We wish to show that corresponding to each elementary column matrix with matrix, say, $E_{k}$, we can find a manipulation of the handle which realizes this change. That is, we want to find a new handle decomposition, equivalent to the one obtained in $S_{m-1}$ (in fact, it will be identical to it on $N(i-1)$ ) such that if $\left\{c_{j}^{i \prime}\right\}$ is the new basis of $C_{i}$ determined by this new decomposition, that the 
matrix relating $\left\{c_{j}^{i{ }^{\prime}}\right\}$ to $\left\{e_{j}^{i}\right\}$ will be the matrix $E A$. If we can do this successively to each $E_{k}, k=1, \cdots, w$, we will finally obtain a new handle decomposition whose handles correspond to a basis $\left\{c_{j}^{i \prime \prime}\right\}$ of $C_{i}$ and such that the matrix relating the basis $\left\{c_{j}^{i \prime}\right\}$ to $\left\{e_{j}^{i}\right\}$ is $I$. This means we will have found a handle decomposition such that the cells of $C_{i}$ corresponding to the handles are $\left\{d_{j}^{i}\right\} \cup\left\{z_{j}^{i}\right\}$. We will then denote the handles corresponding to the $d_{j}^{i}$ by $k_{j}^{i}$ and those corresponding to $z_{j}^{i}$ by $b_{j}^{i}$ and then $b_{1}^{i-1}+\cdots+b_{s_{i-1}}^{i-1}+k_{1}^{i}+\cdots+k_{s_{i-1}}^{i}$ will be an $b$-decomposition since we have $\partial d_{j}^{i}=z_{j}^{i-1}$.

We consider the two types of elementary column operations:

Type I. Adding one column to another.

Type II. Multiplying one column by a nonzero integer.

Operations of Type I are done by using Lemma 1.4 of Barden [1] or the corresponding operations in the proof of Lemma 2 of Kervaire [6]; here, however, we need $i<n-1$.

Operations of Type II are also done by the Lemma 1.4 of Barden except for multiplication by -1 . But this simply amounts to changing one's mind on how to pick an orientation for the cells in the associated $\mathrm{CW}$ complex.

The argument for $S_{m}$ with $m=n-1$ involves the same sort of argument as above using the dual decomposition. $S_{n-1}$ will not be needed in our application of this theorem, only $S_{n-2^{*}}$

Lemma 2.10. If $N^{n} \supseteq$ Int $M^{n}$ and $H=\widehat{M-N}$ and if the bandle decomposition of $M$ rel $N$ bas bandles of type $k$ or less, then the inclusion map induces isomorphisms $\pi_{i}(\partial M) \approx \pi_{i}(H)$ for $i<n-k-1$.

Proof. This is essentially Corollary 12.3 of Mazur [10].

3. Proofs of main theorems. The following construction of the space $N^{*}$ is fundamental in the theorems which are to follow. The conditions we need for this construction will be denoted by "Hypotheses (*)"; and are as follows:

Hypotheses (*). Suppose $M^{n} \supseteq N^{n}$ with $H_{*}(M, N)=0$, and that $M$ has a handle decomposition on $N$ with handles of type less than or equal to $n-2$, where $n=\operatorname{dim} M, n>5$.

First we use Theorem 2.10 and write $M$ as $N$ plus the sum of $b$-decompositions of type $(n-3, n-2)$ or less. That is, we may write

$$
\begin{aligned}
M= & N+b_{1}^{1}+\cdots+b_{s_{1}}^{1}+k_{1}^{2}+\cdots+k_{s_{1}}^{2}+\cdots \\
& +b_{1}^{n-3}+\cdots+b_{s_{n-3}^{n-3}}+k_{1}^{n-2}+\cdots+k_{s_{n-3}^{n-2}}^{n}
\end{aligned}
$$

Let us consider $N[1]=N+b_{1}^{1}+\cdots+b_{s_{1}}^{1}+k_{1}^{2}+\cdots+k_{s_{1}}^{2}$. Now $\pi_{1}(\partial N[1])$ has finitely many generators, and since $\operatorname{dim} \partial N[1] \geq 4$, these may be represented by 
disjointly embedded circles, $S_{1}, \ldots, S_{m}$; and these circles will have disjoint product neighborhoods $T_{1}, \ldots, T_{m}$, with the following two properties:

Property I. The $T_{i}$ do not contain any points of the 2 -handles $k_{1}^{2}, \cdots, k_{s_{1}}^{2}$.

Property II. None of the $a$-sets from the handles $b_{1}^{2}, \cdots, k_{s_{n-3}-2}^{n-2}$ have points in common with any of the sets $T_{i}$.

Now we will use these $T_{i}$ to attach 2 -handles to $N[1]$; denote these handles by $g_{1}^{2}, \cdots, g_{m}^{2}$. Define $N^{*}=N[1]+g_{1}^{2}+\cdots+g_{m}^{2}$. Note that we have $\pi_{1}\left(\partial N^{*}\right)=$ 0 , since we have killed off the fundamental group of $\partial N[1]$ (Lemma 5.2 of Kervaire and Milnor [8]). By Property I we can consider the handles $g_{1}^{2}, \cdots, g_{m}^{2}$ to be attached to the manifold $N+b_{1}^{1}+\cdots+b_{s_{1}}^{1}$. By Property II and Proposition 2.7 we may define $M^{*}=N^{*}+b_{1}^{2}+\cdots+k_{s_{n-3}}^{n-2}$. Note also that we may consider $M \subseteq M^{*}$.

The corollary of the following lemma is a key step in our embedding theorems.

Lemma 3.1. If Hypotheses $(*)$ bold and if also $\pi_{1}\left(\partial M^{*}\right) \rightarrow \pi_{1}\left(H^{*}\right)$ is an isomorphism (this is equivalent to demanding that $\left.\pi_{1}\left(\partial M^{*}\right)=0\right)$, where we let $H^{*}=\widehat{M^{*}-N^{*}}$; then $H^{*}$ is an $b$-cobordism. (This will be a trivial b-cobordism since $\left.\pi_{1}\left(\partial N^{*}\right)=0.\right)$

Proof. First we note that $\pi_{1}\left(H^{*}\right)=0$. This is true since $\partial N^{*}$ is simply connected, and thus the handles $b_{1}^{2}, \ldots, b_{s_{2}}^{2}$ are homotopically trivially attached as subsets of $\partial N^{*}$. Since the rest of the handles are of type 3 or greater, none of the other handles composing $H^{*}$ change the fundamental group.

Now since $H^{*}$ is built from the sum of $b$-decompositions, it is clear that $H_{*}\left(H^{*}, \partial N^{*}\right)=H_{*}\left(H^{*}, \partial M^{*}\right)=0$. Since we have everything simply connected, $H^{*}$ deforms to either boundary component. Thus $H^{*}$ is a (trivial) $b$-cobordism.

Corollary 3.2. If we assume Hypotheses $(*)$ with $\pi_{1}\left(\partial M^{*}\right)=0$ (or equivalently $\pi_{1}\left(\partial M^{*}\right) \rightarrow \pi_{1}\left(H^{*}\right)$ is an isomorphism onto) and we somehow find a smooth embedding of $N^{*}$ in some manifold $Q$, then $M^{*}$, and therefore the submanifold $M \subseteq M^{*}$, will smootbly embed in $Q$.

Lemma 3.3. If $N^{\prime}=N^{n}+b_{1}^{1}+\cdots+b_{s}^{1}$ is $N^{n}$ plus some 1 -handles, $n \geq 3$, and if we are given an embedding $N \subseteq$ Int $Q$ with $q>n$, then we can extend the embedding of $N$ to an embedding of $N^{\prime}$. (We assume bere that the 1-bandles are attacbed on a connected manifold.)

Proof. We may easily find disjoint arcs in $\partial N$ which span the a-spheres (0-spheres) of $b_{i}^{1}$, and these may be pushed out from $N$ into $Q$ so as to obtain a collection of 1 -disks relatively transverse to $\partial N$. There is no problem thickening these into 1 -handles since all the $V_{n, k}$ 's are path connected.

We are now ready to consider the problems of extending an embedding over 
the 2-handles. For the two handles, we will use the obstruction Theorem 2.6 which states that given $N+b^{k+1}$ and an embedding of $N^{n} \subseteq Q^{q}$ we can extend this embedding to an embedding of $N+b$ if

I. There is a $(k+1)$-disk in $Q$ which spans the $a$-sphere of the handle and which intersects the manifold $N$ only in that $a$-sphere.

II. A certain obstruction vanishes. Since we have $k=$ dimension of the $a$-sphere of $b$, this obstruction will be a homotopy class in $\pi_{k}(V(q-k-1, n-k-1))$ 。 This group will be zero if $(q-k-1)-(n-k-1)>k$; i.e., if $q-n>k$.

If we are to be concerned with 2 -handles, condition II will cause no problem if we assume that $q-n \geq 2$. In Lemma 3.4, we may obtain disjoint spanning 2 -disks by the codimension hypothesis; in Lemma 3.5 we may push the interiors of the 2-disks into $Q \times(0,1]$ so that they miss $N \subseteq Q \times\{0\}$.

Lemma 3.4. If $N^{n} \subseteq Q^{q}$ is a 1 -trivial embedding with $q-n \geq 3$, then $N$ can be extended to an embedding of $N^{*} \subseteq Q$.

Lemma 3.5. If $N^{n} \subseteq Q^{q}$ is a 1-trivial embedding with $q-n \geq 1$, then $N^{*} \subseteq Q \times I, q \geq 5$.

The following lemma is a version of the Whitney separation of spheres lemma. The result of the isotopy is that we will have removed the points of intersection $p$ and $q$ from $S_{a} \cap S_{b}$.

Lemma 3.6. Suppose that $M^{n}=N^{n}+b^{n-3}+b^{n-2}$ with $n>5$ and $\pi_{1}(\partial N)=0$-and thus $\partial\left(N+b^{n-3}\right)$ will be simply connected. Let $S_{a}$ be the sphere of the $(n-2)$-bandle; let $S_{b}$ be the b-sphere for the $(n-3)$-bandle; we will choose a $b$-sphere lying in $\partial N$. We may suppose that $S_{a}$ and $S_{b}$ are trans. verse and thus $S_{a} \cap S_{b}$ consists of a finite number of points; suppose that $p$ and $q$ are two such points with opposite intersection numbers. Then we may perform the following construction:

We can find an arc $\alpha$ in $S_{a}$ from $p$ to $q$ and an arc $\beta$ in $S_{b}$ from $p$ to $q$ so that the circle $\alpha \cup \beta$ will lie in $\partial N$ and sucb that the only points of $s_{a} \cap S_{b}$ which will lie on this curve will be $p$ and $q$. Then we may find a 2-disk, $B$, in $\partial\left(N+b^{n-3}\right)$ such that the boundary of $B$ corresponds to $\alpha \cup \beta$. Sucb a disk may be found so as to enable us to construct the following isotopy. We can find a small neighborbood $W^{\prime}$ of $a$ in $S_{a}$ with a contained in the interior of $W^{\prime}$, and a small neighborbood, $W$, of $B$ in $\partial\left(N+b^{n-3}\right)$ and an isotopy $f_{t}$ of $W^{\prime}$ in $W$ fixed on $\partial W^{\prime}$ such that $f_{1}\left(W^{\prime}\right) \cap S_{b}=\varnothing$.

The construction of $f_{t}$ is essentially the same as the Whitney isotopy as described in Milnor [12, Theorem 6.6]. However, this is not the same removal of pairs of intersection points. The reason is this: The isotopy of the disk $W^{\prime}$ 
given by our lemma will not, in general, give us an isotopy of $S_{a}$, as is usually obtained in versions of Whitney's theorem-we will only obtain an isotopy of part of $S_{a}$. This is due to the particular dimensions involved. The dimension of $S_{a}$ is $n-3$; and the dimension of $S_{b}$ is 2 ; both of these spheres are contained in $\partial\left(N+b^{n-3}\right)$ which is an $(n-1)$-manifold. We will obtain $B$ by using relative general position on the disk which spans $\alpha \cup \beta$ via the hypothesis that $\pi_{1}(\partial N)=$ 0 and $n-1 \geq 5$. $S_{b}$ is a 2 -sphere and, again, since $n-1 \geq 5$ it is easy to make sure that $B \cap S_{b}=\beta$. But the codimension of $S_{a}$ in $\partial\left(N+b^{n-3}\right)$ is 2 and thus, in general, the intersection of $B$ and $S_{a}$ will be zero-dimensional. The effect of this is that after the isotopy $f_{t^{\prime}} f_{1}\left(W^{\prime}\right)$ may intersect $S_{a}-W^{\prime}$ and thus we will not end up with an embedding of $S_{a}$. However, the self-intersections of $S_{a}$ will be of such a nature that we will, later, be able to handle these without difficulty.

We may now apply Lemma 3.6 to homotope the $a$-spheres (and thus the a-sets) of the $i$ th $(n-2)$-handles off all $(n-3)$-handles except for one disk which goes around the $i$ th $(n-3)$-handle.

Lemma 3.7. Suppose that $M=N$ plus an b-decomposition of type $(n-3, n-2)$ with $n>5, \partial N$ simply connected. Let $A_{i}$ and $S_{i}$ denote the a-set and a-sphere, respectively, of the ith $(n-2)$-handle, $k_{i}^{(n-2)}$. For each $A_{i}$ and $(n-3)$-bandle $b_{i}$, choose one component, $Y_{i}$ of $A_{i} \cap \partial b_{i}$; this will be an $(n-1)$ disk and will go right around the bandle $b_{i}$ as in the definition of a nicely bandled decomposition. We will assume that the $(n-3)$-disk $X_{i}=S_{i} \cap Y_{i}$ intersects the b-sphere of $k_{i}^{(n-3)}$ with intersection number 1 ; we may now pair off the rest of the intersection points of $S_{i}$ with each of the b-spheres of the $(n-3)$-bandles so that we may apply Lemma 3.6 to each pair.

There is a set of subdisks $W_{i j}$ such that each $W_{i j} \subseteq S_{i}$ (the $j$-index is an arbitrary ordering of the $W_{i j}$ for fixed $i$ ) and subsets $U_{i j}$ of $A_{i}$ corresponding to $W_{i j} \times D^{2}$, and an isotopy, $G_{t}$ of $\partial N$ such that if we let $Z_{i}=\left(\overline{A_{i}-Y_{i}}-\Sigma_{j} U_{i j}\right) \cup$ $\Sigma_{j} G_{1}\left(U_{i j}\right)$, then $Z_{i} \cap \partial b_{j}=0$ for all $i \neq j$. In other words, if we consider the homotopies, $G_{t}^{i}$, on $A_{i}$ defined as $G_{t}$ on $\Sigma_{j} U_{i j}$ and the identity on the rest of $A_{i}$ then we will have

(1) $G_{1}^{i}\left(A_{i}\right) \cap \partial b_{j}=\varnothing$ if $i \neq j$;

(2) $G_{1}^{i}\left(A_{i}\right) \cap \partial b_{i}=Y_{i}$

The next theorem is the result which will allow us to extend a given embedding over an $b$-decomposition of type $(n-3, n-2)$.

Theorem 3.8. Suppose that $M^{n}=N^{n}$ plus an b-decomposition of type $(n-3, n-2)$ with $n>5$ with $\partial N$ simply connected. If we have an embedding of $N^{n} \subseteq$ Int $Q^{q}$ with $n<q$, then we can extend the embedding of $N$ to an embedding of $M$ in $Q$. 
Proof. Recall the $Z_{i}$ of the previous lemma; these are $(n-1)$-disks with self-intersections. $Z_{i}$ is $A_{i}$ minus $Y_{i}$ after the homotopy which removed cancelling intersection points. Let $T_{i}=G_{1}\left(\overline{S_{i}-X_{i}}\right)$, that is, $T_{i}$ is the part of $G_{1}\left(S_{i}\right)$ which lies in $Z_{i}$. These are $(n-3)$-disks with self-intersections; these self-intersections are due to the isotopies of subdisks $W_{i j}$ of $S_{i}$ used in the deformation disks $D_{i j}$, and are confined entirely in the collection of neighborhoods $W_{i j}^{\prime}$ of $D_{i j}$. Also we note that we do not have any singularities on $\partial W_{i j}$, since the $\partial W_{i j}$ are not moved in the isotopies.

We will now define a subset $T_{i}^{\prime}$ of $\partial N \times I$ which will correspond to $T_{i}$ pushed into this set from the boundary, $\partial N \times(0)$. We will also define a similar set $Z_{i}^{\prime}$ for $Z_{i}$. Then we will define a set which we will call $H_{i}^{\prime}$, which will essentially be a thickening of the set $Z_{i}^{\prime}$ in $\partial N(n-3) \times I$. $T_{i}^{\prime}$ by

Let $C_{i}=X_{i} \cap T_{i} ; C_{i}$ corresponds to $\partial X_{i}$ and also $\partial T_{i}$. Then we define

$$
\begin{aligned}
T_{i}^{\prime}= & C_{i} \times[0,1 / 3] \cup\left(\overline{T_{i}-\sum_{j} W_{i j}}\right) \times(1 / 3) \\
& \cup\left(\sum_{j} \partial W_{i j}\right) \times[1 / 3,2 / 3] \cup\left(\sum_{j} W_{i j}\right) \times(2 / 3) .
\end{aligned}
$$

Let $E_{i}=Y_{i} \cap Z_{i}$. Let $U_{i j}$ be the subset of $Z_{i}$ corresponding to $W_{i j} \times D^{2}$; let $U_{i j}^{\prime}$ be the subset of $Z_{i}$ corresponding to $\partial W_{i j} \times D^{2}$. Now we will define $Z_{i}^{\prime}$ by

$$
\begin{aligned}
Z_{i}^{\prime}= & E_{i} \times[0,1 / 3] \cup\left(\overline{Z_{i}-\overline{\sum_{j} U_{i j}}}\right) \times(1 / 3) \\
& \cup\left(\sum_{j} U_{i j}^{\prime}\right) \times[1 / 3,2 / 3] \cup\left(\sum_{j} U_{i j}\right) \times(2 / 3) .
\end{aligned}
$$

Note that if $p: \partial N \times I \rightarrow \partial N$ is the projection on the first factor, then $p\left(T_{i}^{\prime}\right)=T_{i}$ and $p\left(Z_{i}^{\prime}\right)=Z_{i}$.

We will illustrate these definitions by the following example:

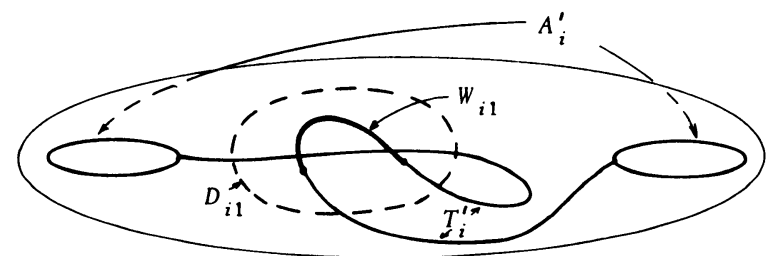


Here $A_{i}^{\prime}$ is the $a$-set of the $i$ th $(n-3)$-handle, $b_{i}$. This example shows a situation with a 1 -handle and a 2 -handle. These are not correct dimensions for our hypothesis, but we will be able to depict our sets with these examples. $T_{i}^{\prime}$ will then look like this:

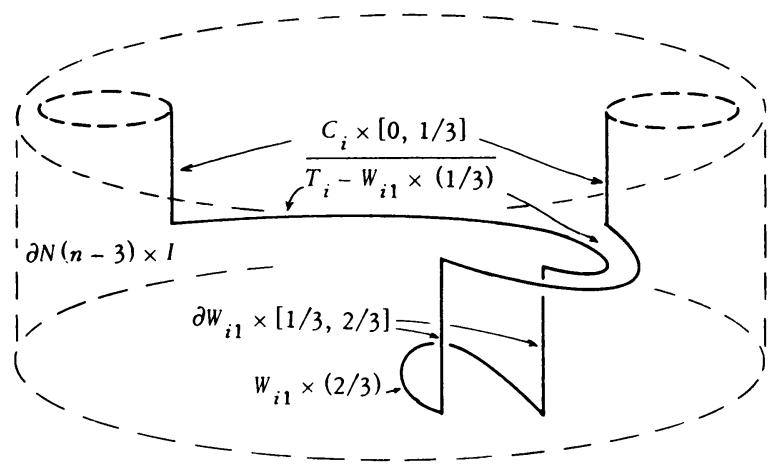

And then $Z_{i}^{\prime}$ will look like this:

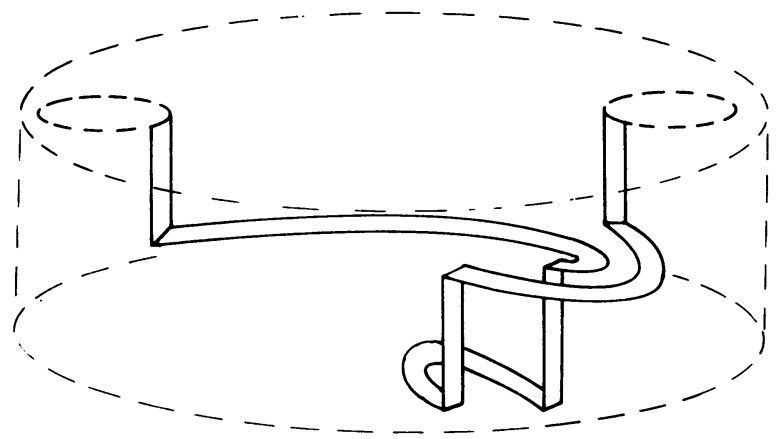

Let $R_{i j}$ be a small collar neighborhood of $U_{i j}$ in $Z_{i}$. We will define a subset $H_{i}^{\prime}$ of $\partial N \times I$ by

$$
\begin{aligned}
H_{i}^{\prime}=A_{i}^{\prime} \times[0,1 / 2] \cup\left(\overline{Z_{i}-\sum_{j} U_{i j}}\right) \times[1 / 3,1 / 2] \\
\cup\left(\sum_{j} R_{i j}\right) \times[1 / 2,1] \cup\left(\sum_{j} U_{i j}\right) \times[2 / 3,1] .
\end{aligned}
$$

Now $H_{i}^{\prime}$ is an $n$-ball in $\partial N \times I$ which intersects $\partial N \times(0)$ in $A_{i}^{\prime}$. Furthermore, $Z_{i}^{\prime}$ lies on the boundary of this ball. Thus $H_{i}^{\prime}$ is just like the handle $b_{i}$, but it is inside $N$ rather than outside: 


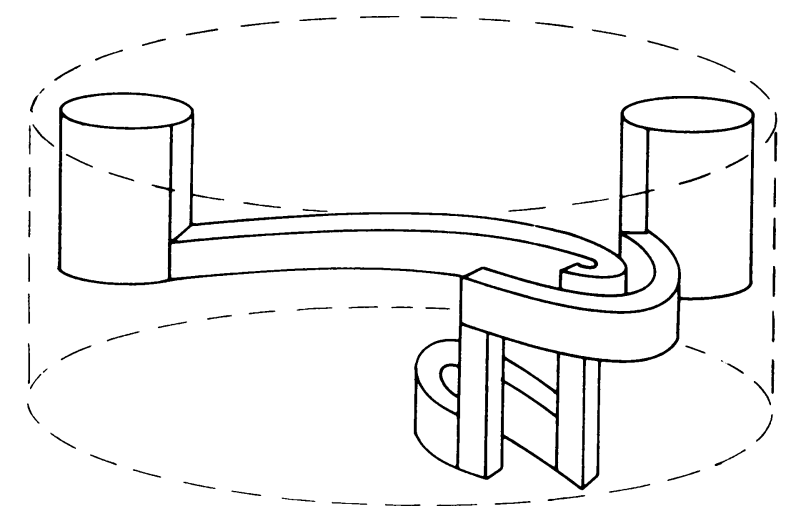

Now let $J=[-1,0]$. We are given an embedding $N \subseteq Q$. Let $N^{\prime}=N+$ $(\partial N \times J)$, where we identify $\partial N$ with $\partial N \times(0)$; then we can extend the embedding of $N$ to an embedding of $N^{\prime}$.

We now consider $\partial N \times D^{2}$. Using polar coordinates for $D^{2}$ we will consider $D^{1}=\left\{(r, \theta) \in D^{2}\right.$ with $\theta=0$ or $\left.\theta=\pi\right\}, I=\left\{(r, \theta) \in D^{2}\right.$ with $\left.\theta=0\right\}$ and $J=$ $\left\{(r, \theta) \in D^{2}\right.$ with $\left.\theta=\pi\right\}$. We now define an isotopy $F_{t}$ of $\partial N \times D^{2}$ by rotating half a revolution by means of the disk $F_{t}(x,(r, \theta))=(x,(r, \theta+t \pi))$. Let $H_{i}=F_{1}\left(H_{i}^{\prime}\right)$; these sets will correspond to our handles $b_{i}$.

To see that the $a$-map of $H_{i}$ is the same up to isotopy as the $a$-map of $b_{i}$, we may argue as follows. Write $D^{n}=D^{n-3} \times D^{3}$, then $b_{i}$ is a smooth embedding of $\partial D^{n-3} \times D^{3}$. Suppose we write $D^{n-3} \times D^{3}=D^{n-3} \times D^{2} \times 1$. Then since our handle decomposition is a nicely handled decomposition, if we may think of $C_{i}$ as the 2-disk $C_{i}=\{0\} \times D^{2} \times\{0\}$ then $Y_{i}$ will correspond to $D^{n-3} \times C_{i} \times\{0\} \subseteq$ $\partial b_{i}$. If we let $b_{i}^{\prime}=b_{i} \mid \partial D^{n-3} \times C_{i} \times\{0\}$, then $b_{i}^{\prime}$ determines $b_{i}$ (up to isotopy) as we may see by applying Lemma 2.4 with $P=\partial D^{n-3} \times C_{i}, Q=b_{i}\left(\partial D^{n-3} \times C_{i} \times\{0\}\right)$ and thus the $a$-set of $b_{i}$ corresponds to $Q \times I$. If we consider $H_{i}^{\prime}$ to be a handle attached from the inside to $\partial N$, then the a-map of this handle, call it also $H_{i}^{\prime}$, will similarly be determined on the set corresponding to $C_{i}$ and thus we may have $H_{i}^{\prime}=b_{i} \circ \psi$ where $\psi$ is the orientation reversing diffeomorphism on $\partial D^{n-3} \times D^{3}=\partial D^{n-3} \times D^{2} \times 1$ defined as the cartesian product of the identity on $\partial D^{n-3}$, the identity on $D^{2}$ and the linear orientation reversing map on $I$.

Similarly, if $H_{i}$ denotes the $a$-map of the handle $H_{i}$, we will have $H_{i}=H_{i}^{\prime} \circ \psi$ and so $H_{i}=b_{i} \circ \psi \circ \psi=b_{i}$.

Thus we may define an embedding $\phi: N+b_{1}^{(n-2)}+\cdots+b_{s_{n-2}}^{(n-2)} \rightarrow N^{\prime}$ so that $\phi(N)=N, \phi\left(b_{i}\right)=H_{i}$ and $\phi\left(Y_{i}\right)=Z_{i}^{\prime \prime}$.

Consider the isotopy $F_{t}$ as giving a map $F:(\partial N \times I) \times I \rightarrow \partial N \times D^{2}$; then define $P_{i}=F\left(Z_{i} \times I\right)$. Viewing $\partial N \times D^{2} \approx \partial N \times D^{\prime} \times I, P_{i}$ will be an $n$-ball which will hit $\partial N \times D^{\prime} \times\{0\}$ in $Z_{i}^{\prime} \cup Z_{i}^{\prime \prime}$ where $Z_{i}^{\prime \prime}=F_{1}\left(Z_{i}^{\prime}\right)$. 
We will next use Lemma 3.9 below to obtain corresponding $n$-balls, $V_{i}$ in $\partial N \times D^{\prime} \times\{0\}$ with $Z_{i}^{\prime} \cup Z_{i}^{\prime \prime} \subseteq \partial V_{i}$; we can arrange it so that the collection $\left\{V_{i}\right\}$ is a disjoint collection of balls.

Lemma 3.9. Suppose $A \approx S^{k} \times D^{r}, A \subseteq W^{w}$ with $w-k \geq 3, w>k+r$; then $A$ lies on the boundary of $a(k+r+1)$-disk in $W$ iff $A \times\{0\}$ lies on the boundary of $a(k+r+1)$-disk in $W \times I$. (In the case $r=0$, this is Lemma 1.5 of Haefliger [2].)

Next, we define a homotopy, $\psi_{t}$, of $\partial N \times D^{\prime}$ such that $\psi_{t} \mid Z_{i}^{\prime} \cup Z_{i}^{\prime \prime}$ is an isotopy and $\psi_{1}\left(Z_{i}^{\prime} \cup Z_{i}^{\prime \prime}\right)=\phi\left(A_{i}\right)$. This homotopy will be essentially given by $K_{t}(x, t)=G_{1-t}(x, 1 / t)$ on $\partial N \times I ; K_{t}(x, t)=$ [identity on $\left.\partial N(n-3) \times J\right]$. However, $K_{t} \mid Z_{i}^{\prime} \cup Z_{i}^{\prime \prime}$ will not be an isotopy since $K_{1}$ will collapse subsets of $Z_{i}^{\prime}$ corresponding to $E_{i} \times[0,1 / 3]$ and $\left(\Sigma U_{i j}\right) \times[1 / 3,2 / 3]$. We may avoid this problem by first applying an isotopy that tilts these sets slightly in the $I$ direction so that the restriction of the projection $\partial N \times I \rightarrow \partial N$ to $Z_{i}^{\prime}$ will be a one-to-one map of $Z_{i}^{\prime}$ onto $Z_{i}$. If we now apply $K_{t}$, we will obtain our $\psi_{t}$.

Next we may extend the isotopies $\psi_{t} \mid Z_{i}^{\prime} \cup Z_{i}^{\prime \prime}$ to isotopies $\overline{\psi_{t}}$ of the $n$-balls $V_{i}$; let $V_{i}^{*}=\overline{\psi_{1}}\left(V_{i}\right)$. Then $\left\{V_{i}^{*}\right\}$ is a disjoint collection of $n$-balls in $N^{\prime}$, and the restriction of the normal bundle of $N^{\prime}$ in $Q$ to $\Sigma V_{i}^{*}$ is trivial; thus we may extend the embedding of $N^{\prime}$ to an embedding of $X=N^{\prime}+\Sigma V_{i}^{*} \times I$.

Let $K_{i}$ be the $n$-ball of $X$ corresponding to $A_{i} \times I \cup V_{i}^{*} \times\{1\}$. These $K_{i}$ will be our $(n-2)$-handles. We may check from our construction that the $a$-map of $K_{i}$, call it $K_{i}$, is the same as $k_{i}$. However, it is easier to argue that these maps are isotopic as follows. The $a$-spheres of these handles are clearly the same, so we may view $K_{i}$ and $k_{i}$ as giving two framings by 2 -frames of this $(n-3)$-sphere. These framings determine elements of $\pi_{n-3}\left(\mathrm{SO}_{2}\right)$ and the framings will be equivalent (and thus $K_{i}$ and $k_{i}$ isotopic) iff they determine the same element; which they must since, in fact, $\pi_{n-3}\left(\mathrm{SO}_{2}\right)=0$ if $n \geq 5$. Thus the desired embedding of $M$ is given by $N+H_{1}+\cdots+H_{s}+K_{1}+K_{2}+\cdots+K_{s}$.

Proof of Theorem A. We begin by considering some handle decomposition of $M$ on $N$. Since $M$ has nonempty, connected boundary, we may assume that this decomposition has no $n$-handles.

We will eliminate the problem of having handles of type one less than the dimension of the manifold by considering $M^{\prime}=M \times I$, and $N^{\prime}=N \times I$. Let $m=\operatorname{Dim} M^{\prime}=n+1$. By Lemma 2.1 we can get a handle decomposition of $M^{\prime}$ on $N^{\prime}$ where the largest type handle is of type $n-1=m-2$; so $M^{\prime}$ has a handle decomposition on $N^{\prime}$ with no $m$-handles and no $(m-1)$-handles.

Since we can embed $N$ in $Q$, we certainly can embed $N \times I$ in $Q \times I$. Now we consider $\left(N^{\prime}\right)^{*}$. By Lemma 3.5 we can embed $\left(N^{\prime}\right)^{*}$ in $(Q \times I) \times I$. 
Now let $M^{\prime \prime}=\left(M^{\prime}\right)^{*}((n-4))$; this will be all of the decomposition of $M^{\prime}$ on $N^{\prime}$ except the last $b$-decomposition of type $(n-3, n-2)$. Now we may use Lemma 3.2 on $M^{\prime \prime}$, since Lemma 2.10 assures us of the condition on the fundamental groups; therefore we can embed $M^{\prime \prime}$ in $Q \times I^{2}$. Finally we use Theorem 3.8 to get an embedding of all of $\left(M^{\prime}\right)^{*}$ in $Q \times I^{2}$. But then we have $M^{\prime}=M \times I \subseteq Q \times I^{2}$.

Proof of Theorem B. We will define $N^{\prime}$ and $M^{\prime}$ as in the previous theorem. We are given $N^{\prime} \subseteq Q$; this time we will use Lemma 3.4 to embed $\left(N^{\prime}\right)$ in $Q$.

We define $M^{\prime \prime}$ as in the previous theorem, and the same argument will show us that we can embed $M^{\prime \prime}$ in $Q$, and thus we can embed $M^{\prime} \subseteq M \times I$ in $Q$.

Proofs of Theorems $A^{\prime}$ and $B^{\prime}$. If we now proceed with the proofs of the previous theorems, using $M$ and $N$ instead of $M^{\prime}$ and $N^{\prime}$, and assuming $n>5$, we could conclude in Theorem $A$ that we could embed $M$ in $Q \times I$; and we could conclude in Theorem B that if $N \subseteq Q$, then $M \subseteq Q$.

4. Embedding homology connected manifolds. A homology cobordism is a triple $\left(W ; M_{0}, M_{1}\right)$ where $\partial W$ is the disjoint union of $M_{0}$ and $M_{1}$ and $H_{*}\left(W, M_{0}\right)=$ $H_{*}\left(W, M_{1}\right)=0$. If $M_{0}$ is a manifold with boundary, a homology cobordism of $M_{0}$ is a 4-tuple $\left(W ; H, M_{0}, M_{1}\right)$ where $\partial W=H \cup M_{0} \cup M_{1}, H \cap M_{0}=\partial M_{0}, H \cap M_{1}=$ $\partial M_{1}, H_{*}\left(W, M_{0}\right)=H_{*}\left(W, M_{1}\right)=0$ and $\left(H, \partial M_{0}, \partial M_{1}\right)$ is a homology cobordism as above.

In the theorem below, we will assume for convenience only that $M_{0}$ has no boundary. If $M_{0}$ has boundary then the homology cobordism we would obtain in the proof of the theorem would be a 4-tuple $\left(W ; H, M_{0}, M_{1}\right)$ with $H \approx \partial M_{0} \times I$.

Theorem 4.1. Let $M_{0}^{n}$ be a smooth compact, orientable manifold $n \geq 5$, with $H_{i}\left(M_{0}^{n}\right)=0,1 \leq i \leq k$, then there is a bomology cobordism $\left(W ; M_{0}, M_{1}\right)$ such that $M_{1}$ is k-connected.

Furtbermore, if $M_{0}$ is a $\pi$-manifold, so is $M_{1}$; if $M_{0}$ is almost parallelizable, so is $M_{1}$.

Proof. As in the proof of Theorem 2.9, we may find a handle decomposition of $M^{0}$ of the following form:

$$
M_{0}=b_{1}^{0}+b_{1}^{1}+\cdots+b_{r}^{1}+k_{1}^{2}+\cdots+k_{r}^{2}+\mathcal{H}
$$

where $\mathcal{H}$ is the sum of handles of type two or greater and $b_{1}^{0}+\cdots+k_{r}^{2}$ is an $b$-decomposition of type $(1,2)$. Let $N_{0}=b_{1}^{0}+\cdots+k_{r}^{2}$, then $\widetilde{H}_{*}\left(N_{0}\right)=0$. Also, the map $\pi_{1}\left(N_{0}\right) \rightarrow \pi_{1}\left(M_{0}\right)$ induced by inclusion is onto since every loop in $M_{0}$ can be represented by a loop in $b_{1}^{0}+\cdots+b_{r}^{1}$.

Thus $N_{0}$ is a smooth homology disk and since all obstructions to trivializing the tangent bundle plus a trivial line bundle vanish, $N_{0}$ is a $\pi$-manifold. Now $\pi_{1}\left(N_{0}\right)$ has $k$ generators; each may be represented by an embedded circle with a 
product normal bundle. We may attach 2-handles to $M_{0} \times I$ along these circles considered as subsets of $N_{0} \times\{1\} \subseteq M_{0} \times\{1\}$; call the resulting manifold $W^{\prime}$, then $W^{\prime}$ will be a cobordism between $M_{0}$ (corresponding to $M_{0} \times\{0\}$ ) and another manifold which we will denote by $M^{\prime}$. We will let $V^{\prime}$ denote the subset of $W^{\prime}$ corresponding to $N_{0} \times I$ plus those 2 -handles; $V^{\prime}$ will be a cobordism between $N_{0}$ and another manifold, denoted $N^{\prime}$. By a proper choice of trivializations of the product bundles, we may add our handles in such a way as to have $N^{\prime}$ a $\pi$-manifold [5, Theorem 5.5].

Also, from the same theorem, we may conclude that $N^{\prime}$ is simply connected. Since $\pi_{1}\left(N_{0}\right) \rightarrow \pi_{1}\left(M_{0}\right)$ was onto, we will then have $M^{\prime}$ simply connected.

Now $H_{2}\left(W^{\prime}, M_{0}\right)=H_{2}\left(V^{\prime}, N_{0}\right)$ is free on $k$ generators; we will now show that each of these can be represented by an embedded 2 -sphere in $N$ ! We consider the exact sequence

$$
H_{2}\left(V^{\prime}\right) \stackrel{j_{*}}{\longrightarrow} H_{2}\left(V^{\prime}, N_{0}\right) \longrightarrow H_{1}\left(N_{0}\right) .
$$

Since $H_{1}\left(N_{0}\right)=0, j_{*}: H_{2}\left(V^{\prime}\right) \rightarrow H_{2}\left(V^{\prime}, N_{0}\right)$ is onto. Viewing $V^{\prime}$ as a cobordism obtained by adding handles to $N^{\prime} \times I$ we see these must be $(n-2)$-handles. Since $n \geq 5$, these are handles of type 3 or greater, thus adding them to $N^{\prime} \times I$ does not introduce any (nontrivial) relative 2-cycles and so $H_{2}\left(V^{\prime}, N^{\prime}\right)=0$; thus we obtain an onto map $i_{*}: H_{2}\left(N^{\prime}\right) \rightarrow H_{2}\left(V^{\prime}\right)$ via the exact sequence

$$
H_{2}\left(N^{\prime}\right) \stackrel{i}{*} \stackrel{*}{\rightarrow}_{2} H_{2}\left(V^{\prime}\right) \longrightarrow H_{2}\left(V^{\prime}, N^{\prime}\right) \text {. }
$$

Thus, via $i_{*} \circ j_{*}$, any element of $H_{2}\left(V^{\prime}, N_{0}^{\prime}\right)$ can be represented by an element of $H_{2}\left(N^{\prime}\right)$. But since $N^{\prime}$ is simply connected, we have $\pi_{2}\left(N^{\prime}\right) \approx H_{2}\left(N^{\prime}\right)$; and since $n \geq 5$ any element of $\pi_{2}\left(N^{\prime}\right)$ may be represented by an embedded 2-sphere. Since $N^{\prime}$ is a $\pi$-manifold of dimension larger than 5, the 2-spheres will have trivial normal bundle (Lemma 5.3 of [5]). We will now add to $N^{\prime} k$ 3-handles, one for each generator of $H_{2}\left(V^{\prime}, N_{0}\right)$ so that if we let $V$ denote $V^{\prime}$ plus these 3-handles, then $V$ will be a cobordism between $N$ and, say, $N_{1}$. Also, since $N^{\prime} \subseteq M^{\prime}$, we may consider these handles as being added to $W^{\prime}$; if we do, the resulting manifold will be called $W$ and will be a cobordism between $M_{0}$ and a manifold $M_{1} \cdot M_{1}$ will be simply connected: since it is obtained from $M^{\prime}$ by adding 3-handles; $M^{\prime}$ was simply connected and the process of adding a 3-handle does not affect the fundamental group.

We next claim that $\left(W ; M_{0}, M_{1}\right)$ is a homology cobordism. By duality, it is sufficient to show $H_{*}\left(W, M_{0}\right)=0$. The skeletal chain complex $\quad\left(W, M_{0}\right)$ is zero except in dimensions 2 and 3 where in each case it is free on $\because$ nerators, one for each handle. By our construction, the boundary map will $g$ : isomorphism between the 3-chains and the 2-chains since it gives a 1-1 co. Idence on the generators; thus $C_{*}\left(W, M_{0}\right)$ is acyclic and $H_{*}\left(W, M_{0}\right)=0$. 
Now by duality [11], we will also have $H_{*}\left(W, M_{1}\right)=0$. From the exact sequences of the pairs, we see that if $M_{0}$ is homology $k$-connected, so is $M_{1}$. But since $M_{1}$ is simply connected, it must be (homotopy) $k$-connected.

To prove the second assertion of the theorem, we note that our surgery was performed in such a way that if $M_{0}$ were a $\pi$-manifold then so would be $M_{1}$; if $M_{0}$ were almost parallelizable, so would be $M_{1}$.

Many embedding theorems assume that one has a $k$-connected manifold. The theorem of this paper in conjunction with theorems of [5] allow us to use some of these theorems to obtain results on embedding homology $k$-connected manifolds, such as the following

Corollary 4.2. If $M_{0}^{n}$ is a smooth compact bomology k-connected manifold, $k \geq 1, n \geq 5$, then $M_{0}^{n}$ embeds smootbly in $R^{2 n-k+1}$.

Proof. By Theorem 4.1 there is a smooth homology cobordism $\left(W ; M_{0}, M_{1}\right)$ with $M_{1}$ a $k$-connected manifold. Let $C$ denote a collar neighborhood of $M_{1}$ in $W ; C \approx M_{1} \times I$. In the proof of the theorem we have seen that $W$ will have a handle decomposition relative to $M_{1}$ consisting of handles of type three or greater, thus $W$ will be simply connected and thus the map $\pi_{1}\left(M_{0}\right) \rightarrow \pi_{1}(\overline{W-C})$ induced by inclusion will be added onto the map and we may use Theorem $\mathrm{B}^{\prime}$. By [3], since $M_{1}$ is $k$-connected, $M_{1}$ embeds in $R^{2 n-k}$, thus $C$ embeds in $R^{2 n-k+1}$.

Remarks. The hypotheses and conclusion of the above corollary are weaker than those of Theorem E of [4]. It is also interesting to note in view of the particular handle decomposition of $W$ relative to $M_{1}$, we are essentially making direct use of Theorem 3.8.

The author thanks the referee for pointing out errors in the original manuscript.

\section{REFERENCES}

1. D. Barden, Structure of manifolds, Mimeographed at Cambridge University, 1964.

2. A. Haefliger, Differentiable embeddings of $S^{n}$ in $S^{n+q}$ for $q>2$, Ann. of Math. (2) 83 (1966), 402-436. MR $34 \# 2024$.

3. A. Haefliger and M. W. Hirsch, On the existence and classification of differentiable embeddings, Topology 2 (1963), 129-135. MR 26 \#6981.

4. M. W. Hirsch, Embeddings and compressions of polyhedra and smooth manifolds, Topology 4 (1966), 361-369. MR 32 \#6478.

5. M. A. Kervaire and J. W. Milnor, Groups of homotopy spheres. I, Ann. of Math. (2) 77 (1963), 504-537. MR 26 \#5584.

6. M. A. Kervaire, Le théorème de Barden-Mazur-Stallings, Comment. Math. Helv. 40 (1965), 31-42. MR 32 \#6475.

7. - On higher dimensional knots, Differential and Combinatorial Topology (A Sympos. in Honor of Marston Morse), Princeton Univ. Press, Princeton, N. J., 1965, pp. 105-119. MR $31 \# 2732$. 
8. M. A. Kervaire and J. W. Milnor, Groups of homotopy spheres. I, Ann. of Math. (2) 77 (1963), 504-537. MR 26 \#5584.

9. B. C. Mazur, A note on some contractible 4-manifolds, Ann. of Math. (2) 73 (1961), 221-228. MR 23 \#A2873.

10. Differential topology from the point of view of simple homotopy theory, Inst. Hautes Études Sci. Publ. Math. No. 15 (1963), 93 pp. MR 28 \#4550.

11. J. W. Milnor, A duality theorem for Reidemeister torsion, Ann. of Math. (2) 76 (1962), 137-147. MR 25 \#526.

12. - Lectures on the h-cobordism theorem, Princeton Univ. Press, Princeton, N. J., 1965. MR 32 \#8352.

13. J. R. Stallings, On infinite processes leading to differentiability in the complement of a point, Differentiable and Combinatorial Topology (A Sympos. in Honor of Marston Morse), Princeton Univ. Press, Princeton, N. J., 1965, pp. 245-254. MR 31 \#5213.

14. S. Smale, Generalized Poincaré's conjecture in dimensions greater than four, Ann. of Math. (2) 74 (1961), 391-406. MR 25 \#580.

15. N. E. Steenrod, The topology of fibre bundles, Princeton Math. Series, vol. 14, Princeton Univ. Press, Princeton, N. J., 1951. MR 12, 522.

DEPARTMENT OF MATHEMATICS, UNIVERSITY OF IOWA, IOWA CITY, IOWA 52240 